\title{
HEME OXYGENASE-1 EXPRESSION IN BRAIN AND LUNGS IN HYPOBARIC HYPOXIA EXPOSED RABBITS
}

\author{
K. Hristov ${ }^{1 *}$, G. Bekyarova ${ }^{1}$, M. Tzaneva ${ }^{2}$ \\ ${ }^{1}$ Division of Pathophysiology, Medical University, Varna, Bulgaria \\ ${ }^{2}$ Department of General Pathology, Medical University, Varna, Bulgaria
}

\begin{abstract}
Exposure to high altitude (and thus hypobaric hypoxia) induces electrophysiological, metabolic, and morphological modifications in the different tissues, leading to oxidative stress and several clinical syndromes. Hypoxia is known to modulate a number of cellular metabolic functions, including what has been called the stress response. One of the stress response proteins is heme oxygenase-1 (HO-1), a ratelimiting enzyme in heme catabolism. It is responsible for converting a potential oxidant, heme, into a potential antioxidant, biliverdin, gaseous carbon monoxide, and free Fe2+. Heme oxygenase cleaves the heme ring at the alpha-methene bridge to form either biliverdin or, if the heme is still attached to a globin, verdoglobin. Biliverdin is subsequently converted to bilirubin by biliverdin reductase. The physiological role of HO-1 induction in hypoxia is still under investigation. It has been proposed that carbon monoxide resulting from HO-1 activity in hypoxic vascular smooth muscle cells plays a role in the regulation of the vessel tone via activation of soluble guanylyl cyclase. Using immunocytochemistry, this study demonstrates the developmental changes of HO-1 protein expression in brain and lungs endothelia from rabbits exposed to severe hypobaric hypoxia in a hypobaric chamber. The results of the study suggest that acute high-altitude hypoxia may serve as a model for oxidative stress and will aid in better understanding and management of hypoxia-induced pathologies.
\end{abstract}

Key words: Heme oxygenase-1, oxidative stress, Hypobaric hypoxia;

\section{INTRODUCTION}

Exercise and high altitude exposure, such as that associated with mountaineering itself, very often result in oxidative damage (Radak et al., 2001; Wozniak et al., 2001). The lower partial pressure of oxygen at high altitudes results in compromised oxygen supply to various tissues. This condition of hypobaric hypoxia combined with serious physical exercises can lead to several neurological clinical syndromes including acute mountain sickness (AMS), coma, seizures, high altitude cerebral edema (HACE), neurological disabilities, cognitive impairment and decreased mental proficiency including decreases judgment, memory, and the performance of discrete motor movements. As the access to mountains have become easier through aerial route and demand for adventure holiday has increased, millions of people now travel to high altitudes for ski, trek, climb, work and thus exposed to deleterious effects of hypobaric hypoxia.

\footnotetext{
*Correspondence to: Krasimir Hristov, Bulgaria, 9002, Varna, 55 Marin Drinov str,IVth floor;Phone +359886850 36, E-mail etlina@gmail.com
}

Hypobaric hypoxia (low partial pressure of oxygen) itself is two bladed possible situation. Firstly it is an usual result of approximately all

high altitude incidents. Secondly it is a modern, still not well studied healing procedure, offered to stimulate and train nonspecific reactivity of the organism. Mechanisms of action for hypobaric hypoxia (HBO) are based on decreasing of both the partial pressure of oxygen and hydrostatic pressure. Decrementing the hydrostatic pressure decreases partial pressure of gases and causes a reduction in the volume of gas-filled spaces according to Boyle's law.

Formation of reactive oxygen species (ROS) and reactive nitrogen species (RNS) is a consequence of aerobic metabolism, since a number of RONS generating systems are created in the body. Indeed, RONS are natural and physiological modulators of the cellular redox milieu and thereby signal controlling factors of a wide range of known and unknown physiological and patho-physiological processes. Despite the multi line antioxidant 
system, the level of RONS generation can exceed the capability of the defense network, leading to oxidative stress (Askew, 2002).

It has been well demonstrated that the increased production of mitochondrial ROS is typical result of an increased oxygen supply. Furthermore, it has been suggested that 1-2\% of the oxygen which enters the mitochondrion, is released as a ROS. On the other hand, it appears that hypoxia can lead to reductive stress, which also results in increased ROS production by the mitochondrial electron transport system (Mohanraj et al., 1998). It is believed that ROS are generated at complex I and complex III of the electron transport chain. During hypoxia, less $\mathrm{O}_{2}$ is available to be reduced to $\mathrm{H}_{2} \mathrm{O}$ at cytochrome oxidase, thus causing accumulation of reducing equivalents within the mitochondrial respiratory sequence. This accumulation is known as reductive stress and this reaction leads to ROS formation by the auto-oxidation of one or more mitochondrial complexes, such as the ubiquinone-ubiquinol redox couple. Khan and O'Brien (1995) previously demonstrated increases in the cellular NADH/NAD + ratio during hypoxia associated reductive stress.

When cells are exposed to various stressors they induced number of so-called stress proteins in order to protect themselves [Barton SGRG et al 2003]. Heme oxygenase-1 (HO-1) known as heat shock protein 32 (HSP32) is one of these proteins.

Heme oxygenase, an essential enzyme in heme catabolism, cleaves heme to form biliverdin, which is subsequently converted to bilirubin by biliverdin reductase, and carbon monoxide, a putative neurotransmitter. Heme oxygenase activity is induced by its substrate heme and by various nonheme substances. Heme oxygenase occurs as 2 isozymes, an inducible heme oxygenase 1 and a constitutive heme oxygenase-2. HMOX1 and HMOX2 belong to the heme oxygenase family. HO-1 may be induced by a variety of seemingly disparate stimuli hyperoxia, hypoxia, ischemia, inflammation, most of which are linked by their ability to provoke oxidative stress [Applegate LA et al 1991, Ryter SW et al 2006]. These stimuli are leading to endothelial dysfunction too.

In connection with the above we decided to investigate the expression of HO-1 in the brain and lungs under pure hypobaric hypoxia conditions (in hypobaric chamber) excluding the influence of physical exercises.

\section{MATERIALS AND METHODS}

We tested the hypothesis that HO-1 is upregulated in the brain and in the lungs in acute hypoxia by exposing male breed less rabbits to 8,000 meters above sea level for 45 minutes (65 hPa of oxygen).

Rabbits were separated in two groups - SHAM group and a group exposed in an altitude chamber, 6 animals in each one. From all the animals were taken portions from brain and lungs in order to research the expression of HO-1 by immunohistochemical analysis. Rabbit's brain and lung specimens were fixed in $10 \%$ neutral buffered formalin and embedded in paraffin. The deparaffinized and rehydrated sections ( $5 \mu \mathrm{m}$ thick) were treated with $1 \%$ hydrogen peroxide for peroxidase activity inhibition for $5 \mathrm{~min}$. Then they were rinsed in $0.1 \mathrm{M}$ phosphate buffered saline (PBS) $(\mathrm{pH} 7.4)$ and treated in normal goat serum for $20 \mathrm{~min}$. Subsequently, the sections were incubated with polyclonal primary antibody for $24 \mathrm{~h}$ at room temperature. Rabbit anti-HO-1antibody (Santa Cruz, USA) was used. After rinsing with PBS the sections were incubated for $20 \mathrm{~min}$ in goat anti-rabbit immunoglobulins at room temperature. Then they were rinsed in PBS again, treated with rabbit peroxidase-anti-peroxidase complex for 20 min at room temperature and then rinsed in PBS. Finally, peroxidase activity was estimated by the diaminobenzydinetetrachloride $\mathrm{H}_{2} \mathrm{O}_{2}$ method. The preparatios were observed on Amploval microscope with software program Zen2012 (Carl Zeiss). The resulting pictures were compared with ImageJ $1.44 \mathrm{p}$ (Wayne Rasband) and statistically tested by the methods of variation analysis

\section{RESULTS}

The received results are giving information about enhancing the expression of HO-1 in endothelial cells for both studied organs after hypobaric hypoxia - brain and lungs.

The results in brain hypoxic tissue a showing enhanced expression of HO-1 $108 \%$ higher than the SHAM one. There is a statistically significant increase with a confidence of $\mathrm{P}$ $<0.01$ and variance coefficient of $15 \%$. With respect to lungs hypoxic tissue the expression of $\mathrm{HO}-1$ is slight $-36 \%$, relatively to the SHAM, a confidence of $\mathrm{P}<0.05$ and variance coefficient of $28 \%$. We consider such situation to be a result of started consumption of adaptive mechanisms in the lungs. The cited results give us reason to suspect the studied level of hypobaric hypoxia to be probably threshold level between compensation and decompensation stages of oxidative stress. 

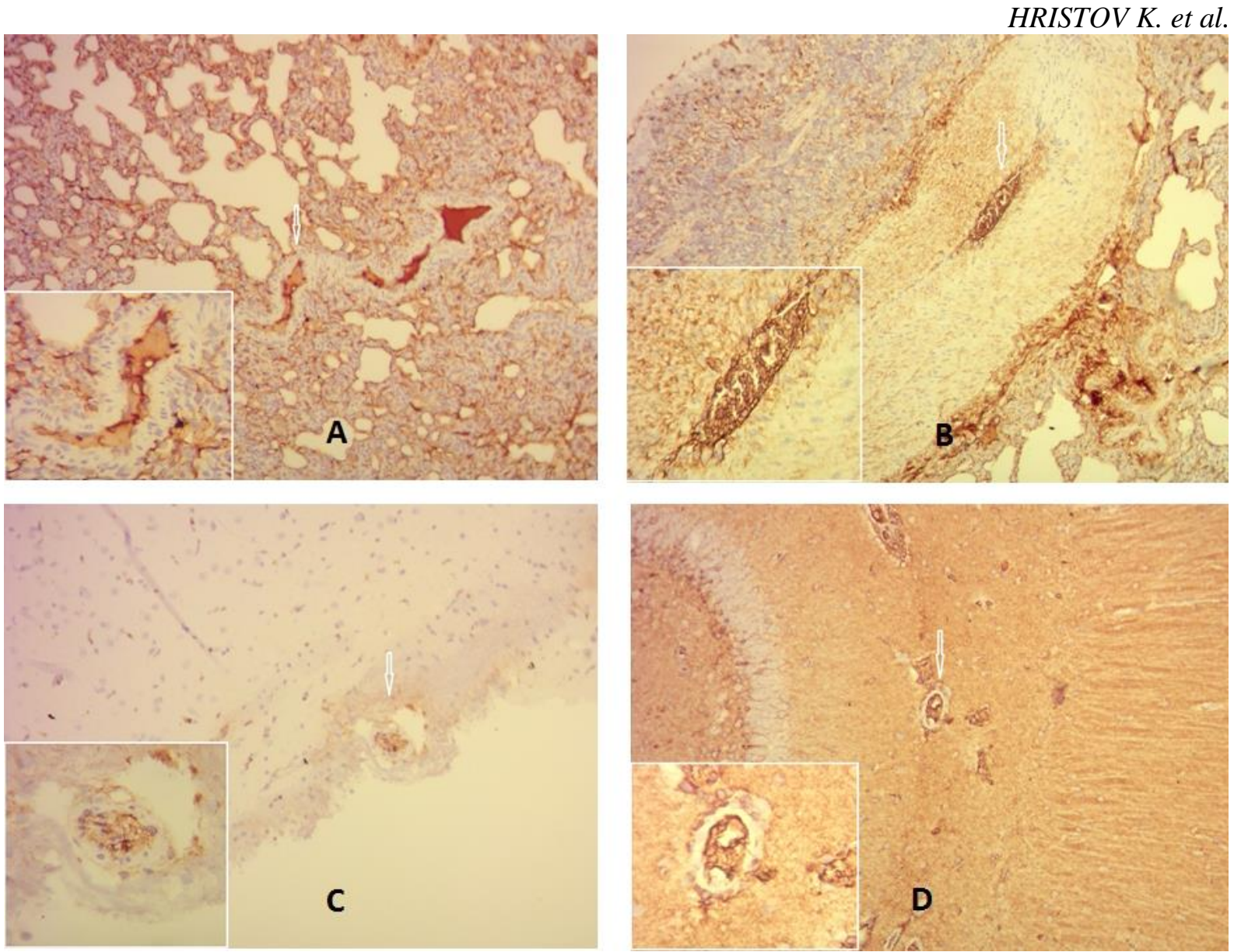

Figure 1. Expression of HO-1 by immunohistochemical analysis in rabbit's brain and lung endothelia.

A - Rabbit's lung control (SHAM); B - Rabbit's lung under hypobaric hypoxia;

C - Rabbit's brain control (SHAM); D - Rabbit's brain under hypobaric hypoxia

(Rabbit's brain and lung specimens were fixed in 10\% neutral buffered formalin and embedded in paraffin. The deparaffinized and rehydrated sections $(5 \mu \mathrm{m}$ thick) were treated with $1 \%$ hydrogen peroxide for peroxidase activity inhibition for $5 \mathrm{~min}$. Then they were rinsed in $0.1 \mathrm{M}$ phosphate buffered saline (PBS) (pH 7.4) and treated in normal goat serum for $20 \mathrm{~min}$. Subsequently, the sections were incubated with polyclonal primary antibody for $24 \mathrm{~h}$ at room temperature. Rabbit anti-HO-1antibody (Santa Cruz, USA) was used.)

\section{DISCUSSION}

Generation of ROS and oxidative stress in the mitochondria are crucial in pathogenesis of hypobaric hypoxia injury. Low leveled oxygen breathing at sub-atmospheric pressure seems to represent a well-suited model for investigating oxidative stress-induced damages as well as protective mechanisms. HO-1 is the inducible isoform of $\mathrm{HO}$, which catalyzes the first and rate-limiting step in heme degradation to produce equimolar quantities of biliverdin, $\mathrm{CO}$ and free iron [Otterbein LE, Choi AM 2000]. Biliverdin is subsequently converted to bilirubin via the action of biliverdin reductase and free iron. Sequestration of free iron by ferritin lowers the prooxidant state of the cell [Sammut IA et al 1998]. Bilirubin and biliverdin are potent antioxidant and $\mathrm{CO}$ has anti-inflammatory and anti-apoptotic effect [Bach FH 2002]. CO stimulates the formation of cyclic guanosine monophosphate and lead to vasodilatation and inhibition of platelet aggregation which could prevent brain and lung low oxygen perfusion disturbances [Kim $\mathrm{HP}$ et al 2006]. Endothelial dysfunction and the impact of the heme-heme oxygenase (HO) system on this process depend on the levels of the heme-dependent generation of reactive oxygen species (ROS) and vasoconstrictor molecules. Induction of HO-1 by its substrate, heme, results in an increase of heme turnover and decreases in ROS and vasoconstrictors generated by emedependent enzyme systems. Decreases in the heme-HO system diminished vasoconstrictors generated by NADPH oxidase (NOX-2)-derived ROS, as well as ROS generated from activation of cyclooxygenase (COX) 1 during arachidonic acid (AA) metabolism by the aorta to prostaglandins. Recent experimental evidence shows that HO1-deficient cells are susceptible to the accumulation of free radicals and to oxidative injury after endotoxin administration [Vile GF, Tyrrel RM 1993]. Therefore, induction of HO1 may provide cytoprotection against oxidative stress. Its expression mainly in the endothelial cells seems to be crucial for protection against oxidative stress damages in the studied organs (brain and lungs). 


\section{CONCLUSION}

The studied level of hypobaric hypoxia as 65 $\mathrm{hPa}$ of oxygen (equal to $8000 \mathrm{~m}$ above the sea level) for $45 \mathrm{~min}$ is probably threshold level between compensation and decompensation stages of oxidative stress. It assumes that lower levels of high altitude conditions (below 8000 $\mathrm{m}$ above the sea) will be better compensated regarding the oxidative stress and confirms the possibility to use the hypobaric hypoxia as healing method which can be used to stimulate and train nonspecific reactivity of the organism.

\section{REFERENCES}

1. Andrei L. Kleschyov, Maria Wendt, Thomas Munzel. Heme Oxygenase-1Mediated Protection: Potential Role of Nonheme Iron Nitric Oxide Complexes. Circulation. 2002;105:e196;

2. Applegate LA, Luscher P, Tyrrell RM. Induction of heme oxygenase: a general response to oxidant stress in cultured mammalian cells. Cancer Res 1991; 51: 974-78.

3. Askew, E.W., 2002. Work at high altitude and oxidative stress: antioxidant nutrients. Toxicology 180, 107-119.

4. Bach FH. Heme oxygenase-1 as a protective gene.Wien Klin Wochenschr 2002; 114, Suppl 4, 1-3.

5. Barton SGRG, Rampton DS, Winrow VR, Domizio P, Feakins RM. Expression of heat shock protein 32 (hemoxygenase-1) in the normal and inflamed human stomach and colon: an immunohistochemical study. Cell Stress \& Chaperones 2003; 8: 329-34.

6. Carraway M. S., Taylor J. L., Piantadosi C. A.(1998) Regulation of heme oxygenase activity by oxygen (Abstract). Undersea Hyperb. Med. 25S:55.

7. Frank L.(1982)Protection from O2 toxicity by preexposure to hypoxia: lung antioxidant enzyme role. J. Appl. Physiol. 53:475482.3.

8. Gozzelino R, Jeney V, Soares MP. Mechanisms of cell protection by heme oxygenase-1. Annu Rev Pharmacol Toxicol. 2010; 50:323-54.
9. Groger M, Speit G, Radermacher P, Muth CM. Interaction of hyperbaric oxygen, nitric oxide, and heme oxygenase on DNA strand breaks in vivo. Mutat Res. 2005 May 2;572(1-2):167-72.

10.Khan, S., O'Brien, P.J., 1995. Modulating hypoxia-induced hepatocyte injury by affecting intracellular redox state. Biochim. Biophys. Acta 1269, 153-161.

11.Kim HP, Ryter SW, Choi AM. CO as a cellular signaling molecule. Annu Rev Pharmacol Toxicol 2006; 46: 411-49.

12.Maines M D. Heme oxygenase: function, multiplicity, regulatory mechanisms, and clinical applications. The FASEB Journal 1988, vol. 2, no.10, 2557-2568;

13.Masaki Unno, Toshitaka Matsuia, Masao Ikeda-Saito. Structure and catalytic mechanism of heme oxygenase. Nat. Prod. Rep., 2007, 24, 553-570;

14.Mohanraj, P., Merola, A.J., Wright, V.P., Clanton, T.L., 1998. Antioxidants protect rat diaphragmatic muscle function under hypoxic conditions. J. Appl. Physiol. 84, 1960-1966.

15. Otterbein LE, Choi AM. Heme oxygenase: colors of defense against cellular stress. Am J Physiol Lung Cell Mol Physiol 2000; 279: L1029-37.

16.Radak, Z., Taylor, A.W., Ohno, H., Goto, S., 2001. Adaptation to exerciseinduced oxidative stress: from muscle to brain. Exerc. Immunol. Rev. 7, 90-107

17. Ryter SW, Alam J, Choi AM. Heme oxygenase-1/carbon monoxide: from basic science to therapeutic applications. Physiol Rev 2006; 86: 583-650.

18. Sammut IA, Foresti R, Clark JE, Exon DJ, Vesely MJ, Sarathchandra P, Green CJ, Motterlini R. Carbon monoxide is a major contributor to the regulation of vascular tone in aortas expressing high levels of haeme oxygenase-1. Br J Pharmacol 1998; 125: $1437-44$.

19.Vile GF, Tyrrell RM. Oxidative stress resulting from ultraviolet $\mathrm{A}$ irradiation of human skin fibroblasts leads to a heme oxygenase-dependent increase in ferritin. J Biol Chem 1993; 268: 14678-81. 